\title{
Improvement of sensorimotor functions in old age by passive sensory stimulation
}

\author{
Tobias Kalisch' \\ Martin Tegenthoff ${ }^{2}$ \\ Hubert R Dinse' \\ 'Department of Theoretical Biology, \\ Institute for Neuroinformatics; \\ 2Department of Neurology, \\ BG-Kliniken Bergmannsheil, \\ Ruhr-University Bochum, Bochum, \\ Germany
}

\begin{abstract}
Sensorimotor functions decrease in old age. The well-documented loss of tactile acuity in elderly is accompanied by deterioration of haptic performance and fine manipulative movements. Physical training and exercise can maintain sensorimotor fitness into high age. However, regular schedules of training require discipline and physical fitness. We here present an alternative interventional paradigm to enhance tactile, haptic, and fine motor performance based on passive, sensory stimulation by means of tactile coactivation. This approach is based on patterned, synchronous tactile stimulation applied to the fingertips for 3 hours. The stimulation drives plastic reorganizational changes in somatosensory cortex that affect perception and behavior: We demonstrate that following 3 hours of coactivation tactile acuity as well as haptic object exploration and fine motor performance are improved for at least 96 hours. Because this kind of intervention does not require active participation or attention of the subjects, we anticipate that coactivation is a prime candidate for future therapeutic interventions in patients with impaired sensorimotor abilities. It can be assumed that the maintenance and restoration of sensorimotor functions can ensure and preserve independence of daily living. Further optimizing of the stimulation protocol can be assumed to strengthen both the range and durability of its efficacy.
\end{abstract}

Keywords: aging, tactile acuity, coactivation, cortical plasticity, intervention, sensorimotor performance

\section{Introduction}

It is common wisdom that physiological processes lose efficiency in old age (Chodzko-Zajko 1997; Young 1997). To preserve and to ensure the ability for appropriate interaction with the environment, a wide range of technical devices is available and utilized to attenuate age-related impairment of the human senses. For example, glasses and hearing aids became a kind of standard aid for elderly people. Surprisingly, in contrast to vision and hearing, the sense of touch is widely ignored and its vital role for coping with activities of daily living is underestimated. To this day there are only few approaches and concepts to counteract impairment of tactile perception in old age, although perceptual and behavioral abilities depend on an intact sense of touch.

For many reasons the human sense of touch and therefore tactile acuity decreases in old age (Thornbury and Mistretta 1981; Stevens 1992; Stevens and Patterson 1995; Stevens and Choo 1996; Stevens and Cruz 1996; Sathian et al 1997; Tremblay et al 2003; Dinse et al 2006). Age-related alterations occur at all levels of the somatosensory pathway. Skin conformance changes in old age (Cua et al 1990), although it was previously shown that the mechanical properties of the glabrous skin might have minor effects on discriminative abilities in elderly (Woodward 1993; Vega-Bermudez and Johnson 2004). Many reports describe an age-dependent morphological change and loss of dermal receptors (Bruce 1980; Cauna 1987; Besne et al 2002; Iwasaki et al 2003). For example, the number and morphology of Meissner's and Pacinian corpuscles
Correspondence: Hubert R Dinse Institute for Neuroinformatics, Dept. Theoretical Biology, Ruhr-University Bochum, D-44780 Bochum, Germany Tel +49234322 5565

Fax +4923432I 4209

Email hubert.dinse@neuroinformatik.rub.de 
change in old age, while Merkel-neurite complexes appear to be less affected (Bolton et al 1966; Quilliam and Ridley 1971). Furthermore, nerve conduction velocity declines with increasing age (Rivner et al 2001; Valerio et al 2004) and action-potential amplitude is reduced (Bouche et al 1993; Caruso et al 1993). There is compelling evidence for changes of morphology of the central nervous system (Raz et al 1997; Sowell et al 2003) and changes of cortical activation patterns in old age (Cabeza et al 2002). Taken together, these age-related alterations affect somatosensory processing in its entirety and therefore also tactile and haptic perception. If these changes develop slowly and are part of a normal, ie, nonpathological aging process over decades, most elderly progressively adapt to the loss of high-level tactile performance and learn to compensate by developing behavioral strategies such as relying stronger on visual control to cope with the challenges of a visually dominated environment.

However, the importance of tactile perception for guiding sensorimotor performance clearly becomes apparent if the availability of cutaneous information is blocked or reduced. Klatzky and Lederman (1993) demonstrated that the human ability to identify objects by a haptic exploration is very powerful and reliable (Klatzky et al 1985; Klatzky and Lederman 1995), but can be easily disturbed by limiting the access to cutaneous information (Lederman and Klatzky 2004). Sensory information is not only crucial for haptic exploration, but for motor performance as well (Tremblay et al 2003; Goodwin and Wheat 2004; Tamburin et al 2004). Especially during fine manipulative operations, motoneurons are constantly tuned by sensory inputs (Evarts and Fromm 1979). If afferent sensory information is suppressed by local anesthesia of peripheral nerves, motor control is severely impaired despite visual control (Johansson and Westling 1984; Monzee et al 2003), supporting the critical role of sensorimotor integration (Johansson and Westling 1987). When objects are manipulated by means of precision grips, the crucial sensory information supposedly comes from superficial dermal receptors rather than from deep kinesthetic receptors (Moberg 1983). Ebied (2003) demonstrated that the impact of sensory information depends on the complexity of a manipulative task. After anesthesia of the median nerve, subjects were still able to maintain grip strength, but failed in complex tasks (Ebied 2003). For example, the accurate positioning of the fingertips on the surface of an object is essential for successful manipulation (Jeannerod et al 1995; Birznieks et al 2001; Gysin et al 2003).

Besides experiments performed in healthy subjects, strong evidence for the importance of sensory tactile information for sensorimotor performance comes from investigation in patients suffering from acute stroke. If tactile sensibility is lost by a purely sensory stroke, related skills like haptic object exploration or fine motor performance are massively affected as well (Smania et al 2003). Furthermore, the rehabilitation of motor skills after stroke is hindered if sensory perception is also affected, pointing to the importance of sensory tactile information and sensorimotor integration for motor rehabilitation (Reding and Potes 1988).

In addition to the effects and consequences arising from aging processes, the individual amount of use, as well as tactile and haptic skills have important implications on sensorimotor performance. Since aging leads to a decrease in fine motor performance (Krampe 2002), elderly often tend to reduce their activities of everyday life step by step. Reduced use is associated with reduced sensory input. As a result, cortical representational maps are degraded and tactile and haptic processing becomes impaired. Conceivably, these cortical age-related changes mark the beginning of a vicious circle, which has a further negative impact on sensorimotor performance.

Consequences of use and disuse of sensorimotor skills on cortical map topography and performance are captured in the concept of use-dependent plasticity and are intensively investigated. Animal studies first demonstrated that cortical maps are enlarged as a result of training (Recanzone et al 1992, 1993; Dinse and Merzenich 2002). These results were confirmed and extended by imaging studies in humans that revealed enlarged cortical representations in human subpopulations (musicians or blind Braille readers) after a long-term training of their respective sensorimotor skills (Pascual-Leone and Torres 1993; Elbert et al 1995a; Pantev et al 1998; Sterr et al 1998b). Furthermore, proportionality was reported between the absolute performance level and the observed cortical changes (Elbert et al 1995b; Buonomano and Merzenich 1998; Dinse and Merzenich 2002). On the contrary, situations of disuse such as limb immobilization during wearing a cast were shown to shrink cortical representations in parallel to behavioral and perceptual impairment, demonstrating that cortical plasticity mechanisms act in conditions of both enforced and reduced use (Liepert et al 1995). A recent study demonstrated that in patients suffering from carpal tunnel syndrome (ie, a pathological compression of median nerve fibers at the wrist) the cortical hand representations underwent major reorganization (Tecchio et al 2002).

Taken together, the age-related loss of tactile perception and reduced practice of sensorimotor skills might finally 
lead to a situation in which independent everyday life is compromised (Williams et al 1982; Falconer et al 1991; Hughes et al 1997; Giampaoli et al 1999).

A potential intervention to prevent age-related decline is active training, which, if applied at higher age, can restore, maintain and even improve sensorimotor functions affected by aging (Pascual-Leone and Torres 1993; Elbert et al 1995a; Hashimoto et al 2004). However, since many elderly suffer from restricted mobility, additional and alternative approaches are needed to supplement and enhance, or even replace conventional training procedures (Verbrugge and Jette 1994; Dinse et al 2005, 2006).

A paradigm that we introduced several years ago constitutes such an alternative approach to training: a specific form of tactile coactivation (CA), ie, a passive stimulation paradigm based on Hebbian synaptic plasticity to drive perceptual learning in young and adults. In this paradigm, synchronous neural activity is evoked by coactivating small skin portions of the tip of the right index finger for a few hours. As a result, the finger representation in somatosensory cortex enlarged and tactile acuity improved (Pleger et al 2001; Dinse et al 2003; Pleger et al 2003). Recently we reported that $\mathrm{CA}$ is highly effective even in elderly subjects (Dinse et al 2006). Following 3 hours of CA the discrimination thresholds of 80-year-old elderly came to match those of subjects 30 years younger. The unique advantage of $\mathrm{CA}$ is its passive nature, ie, it does not require the active cooperation and involvement of the subject. Even more, attention is not required to drive plastic changes implying that $\mathrm{CA}$ can be applied in parallel to other occupations and therefore might be substantially easier to implement and has a higher chance of being accepted as intervention (Bliem and Dinse unpublished). Together with the effectiveness of coactivation in improving tactile perception even in elderly, these properties make coactivation-based principles prime candidates for therapeutic intervention programs that serve as training substitute in impaired populations.

In recent years we further optimized the CA paradigm in several respects. For example, the duration of application was reduced to $20 \mathrm{~min}$ (Ragert et al 2008) and the stimulation was extended from a single finger to all fingers of a hand (Kalisch et al 2007). As described in our previous work (Kalisch et al 2007), multifinger CA is a useful tool to improve tactile spatial acuity in young adults by inducing particular forms of synaptic plasticity processes (Dinse et al 2003, 2005). We here report new findings from elderly subjects to demonstrate the impact of multifinger CA not only on tactile acuity but also on haptic and fine motor skills.

\section{Subjects and methods Subjects}

A total of 22 subjects ( 9 male, 13 female) participated in our study. The target group comprised of 16 subjects (age: $74.4 \pm 5.6$ years) and the control group ("sham") of 6 subjects (age: $73.5 \pm 4.5$ years). There was no significant age-difference between the subjects of both groups (t-test, $p=0.725)$. All subjects were recruited by get-togethers and seminars or poster announcements in retirement homes. Eligibility criteria for participation were lucidity, independence in everyday activities, neurological health, and the absence of sensorimotor handicaps of the upper limbs. All subjects agreed to report their actual and former medication; as a consequence any influence of drugs on their central nervous system was ruled out. Furthermore all subjects performed the Mini-Mental State Examination (MMSE) (Folstein et al 1975), to test for dementia. Only subjects with scores of 27 to 30 out of 30 , indicative of "no dementia", participated in the study (there was no significant difference in MMSE performance between the subjects of both groups; t-test, $p=0.434)$. Accordingly, the subjects included in our study represent a subpopulation clearly biased towards mental and physical fitness. Hand preference was determined with the Edinburgh Handedness Inventory (Oldfield 1971) which classifies handedness on the basis of a short interview on hand preference in the performance of routine practical tasks. Only persons with unambiguous right-hand dominance and without a history of dominant hand change during their childhood were included. All subjects gave their written informed consent, and the protocol was approved by the local ethics committee of the Ruhr-University Bochum.

\section{Touch threshold}

Fine-touch sensitivity was evaluated by probing the fingertips of the right hand with von Frey filaments (Marstocknervtest, Marburg, Germany) to assess touch thresholds following the procedures described with Semmes-Weinstein monofilaments (Thornbury and Mistretta 1981; Bell-Krotoski et al 1995). Each filament was calibrated to a known buckling force determined by its length and diameter. The von Frey test-kit contains 16 different filaments calibrated to forces ranging from $0.25-294 \mathrm{mN}$ in logarithmic scaling. Additionally two filaments with forces of $0.08 \mathrm{mN}$ and $0.20 \mathrm{mN}$ were used to expand the test range (Touch Test, Stoelting Co, Wood Dale, IL, USA). Fine-touch sensitivity was tested with a staircase procedure, during which subjects were required to indicate whenever they perceived an indentation of the skin on their fingertips. Starting with a noticeable stimulus, 
the applied contact forces were decreased in a step-wise manner until the subjects no longer perceived the stimulus (lower boundary) and then increased until the stimulus was perceived again (upper boundary). This procedure was repeated three times, resulting in six values that were averaged to form the touch threshold.

\section{Two-point discrimination threshold}

The two-point discrimination threshold is a reliable marker for tactile acuity in humans (Pleger et al 2001, 2003; Dinse et al 2003, 2005, 2006; Ragert et al 2004; Kalisch et al 2007). Spatial two-point discrimination thresholds were assessed on the tip of the index fingers of the right hand using the method of constant stimuli as described previously (Godde et al 2000; Pleger et al 2001, 2003; Dinse et al 2003, 2005, 2006; Tegenthoff et al 2005). We tested seven pairs of brass needles, in addition zero distance was tested with a single needle. To overcome problems in the use of two-point measurements associated with hand-held probes, we used a specifically designed apparatus that allows a standardized and objective form of testing (compare with figures in Dinse et al 2005, 2006). The apparatus allows a rapid switching between pairs of needles of different separations or the presentation of one single needle that are applied to a fixed position on the skin of the fingertips for approximately 1 second. To extract thresholds, we obtain psychometric curves based on many repeated stimulus presentations. According to own unpublished data, acuity thresholds obtained by gratings or by two-point measurements are highly equivalent (Pearson-correlation, $r=0.716$, $\mathrm{p} \leq 0.001, \mathrm{n}=22$ subjects), although thresholds obtained by gratings are slightly lower in general.

To account for the age-related decline in tactile acuity (Stevens 1992; Woodward 1993; Sathian et al 1997; Tremblay et al 2003; Dinse et al 2005, 2006), we used larger needle separations for the elderly subjects (ie, 1.5, 2.3, $3.1,3.9,4.7,5.6$, and $7.0 \mathrm{~mm}$ ) than usually used for young subjects (ie, $0.7-2.5 \mathrm{~mm}$ ). The diameter of the needles was $0.7 \mathrm{~mm}$ and the diameter of the blunt endings was $200 \mu \mathrm{m}$. Application-force was about 150 to $200 \mathrm{mN}$. Fixation of the test finger prevented subjects from explorative finger movements. As described previously, test-re-test reliability using this procedure was 0.90 for young subjects, and 0.88 for elderly subjects (Dinse et al 2006).

All eight test conditions were presented eight times in randomized order resulting in 64 tests per session. The subjects, who were not informed about the ratio of needle-pairs and single needles (ie, 7:1), had to decide immediately if they had the sensation of one or two needles. Subjects were instructed to classify the perception of a single needle or doubtful stimuli as "one" but the distinct perception of two stimuli as "two". The summed responses were plotted against the needle-distances resulting in a psychometric function, which was fitted by a binary logistic regression (SPSS; SPSS Inc, Chicago, IL, USA). Threshold was taken from the fit where $50 \%$ correct responses were reached. All subjects had to attend two test sessions to become familiar with the testing procedure before the assessment was started in the third session. In addition to the fingers of the right hand that were all coactivated, we additionally tested the left index finger to control for changes in thresholds indicative of a side-to-side transfer of the CA effect.

\section{Mislocalization test}

In the mislocalization test fingers of the hand are stimulated near threshold, which evokes localization errors, ie, a neighboring finger other than the stimulated one is incorrectly perceived as being stimulated. Schweizer and colleagues (2000, 2001) showed that localization errors across fingers obeyed a somatotopic principle where stimuli are preferentially mislocalized to sites adjacent to the stimulated skin region ruling out pure guessing behavior (Craig and Rhodes 1992; Braun et al 2005). In the present study, we used a set of monofilaments $(0.08-294 \mathrm{mN})$ to conduct a five-alternative forced choice detection test on the fingertips of the right hand (for a detailed description of the test, see Schweizer et al 2001). Each finger was stimulated 20 times in randomized order. Each correct response was followed by a stimulation of lower intensity, and each error was followed by a stimulation of higher intensity to the same finger. Using this procedure, the number of mislocalizations was adjusted to $\sim 50 \%$ of all stimuli. Mislocalizations were analyzed according to their distribution on the fingers. In cases where the staircase procedure did not result in 50\% mislocalizations, data was normalized to 10 mislocalizations per finger. To achieve an overview of individual mislocalization behavior, the mislocalizations from each finger to any other finger were categorized as mislocalizations from the stimulated finger to the first-, second-, third-, or fourth neighboring finger leading to a total of eight first-, six second-, four third-, and two fourth neighbor fingers. Mislocalizations from the stimulated finger to neighboring fingers were averaged for each hand, subject, and session (Kalisch et al 2007). As the mislocalization test was applied after the assessment of individual touch thresholds in every session, the respective calculated touch thresholds were used as a starting point for the threshold-near stimulation in the mislocalization test. 


\section{Haptic object recognition test}

The ability to recognize objects by their haptic impression was tested by means of a custom-made visuo-haptic test. The test consisted of five different groups of unfamiliar cubic objects $(1.5 * 2.7 * 4.7 \mathrm{~cm})$ made from common LEGO $^{\text {TM }}$ bricks (Figure 1). In each group, objects consisted of a specific number of rectangular bricks protruding on the sides in various positions. The constructional differences were highlighted in color to facilitate visual discrimination One sample from each group was placed clearly visible on a desk in front of the subject. In a familiarization phase the experimenter introduced the subject to the constructional differences of the objects, and haptic and visual exploration was allowed. Afterwards the subjects were informed about the objective of the test: A total of 17 objects, hidden in a fabric bag, had to be explored by haptic perception only, ie, by explorative hand movements of the right hand. To that end, after haptic exploration without visual guidance and after deciding upon the group the object was assumed to belong to, the object had to be placed in a box behind the specific sample on the desk. No visual verification during this process was allowed. Subjects were instructed to perform the test as fast and as accurate as possible. After one initial training session all subjects indicated good comprehension of the test. Individual performance was assessed by measuring the time to perform the task and by counting the number of errors from three consecutive sessions that were then averaged.

Conceivably, the identification of common objects from every-day life depends massively on top-down information and will therefore be largely based on existing knowledge and former experience rather than manual exploration. Therefore unfamiliar instead of common objects were used to prevent the influence of prior knowledge about structural information and thus to create a comparable situation for all subjects.

\section{Peg-board test}

To test for fine-motor performance we used a peg-board set-up that is part of a commercial test-battery (MLS,
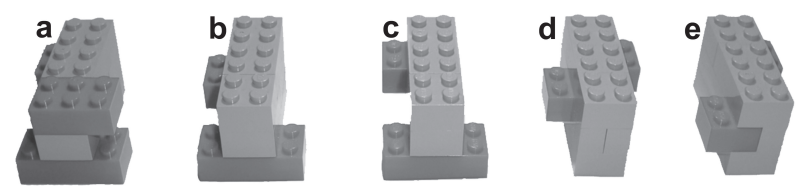

Figure I A set of five groups of unfamiliar objects made from LEGO ${ }^{\text {TM }}$ bricks was used for the haptic object recognition test. In each group, the objects consisted of a cuboid $(1.5 * 2.7 * 4.7 \mathrm{~cm})$ with a specific number and position of rectangular structures on the sides. A total of 17 objects $(3 * a, 5 * b, 4 * c, 3 * d, 2 *$ e) was used for the test. All objects had to be allocated to samples of the five groups $(\mathbf{a}-\mathbf{e})$ only by explorative movements of the dominant hand and without visual verification.
Dr. G. Schuhfried GmbH, Mödling, Austria). The square pegboard $(30 * 30 \mathrm{~cm})$ carries two rows of 25 small holes, one on the left side and one on the right side. Two containers, each equipped with 25 metal pins, were placed in $30 \mathrm{~cm}$ distance from the right and left side of the board. The subjects were asked to pick the pins with their right hand, one by one, from a container and insert them into the holes on the peg-board. If one of the metal pins dropped during the transfer, they were instructed to go on with the next one. During the test the experimenter measured the time to complete the test and additionally the number of dropped pins. The test was performed in standard version (metal pins were $5 * 0.25 \mathrm{~cm}$ ) and in a more demanding version (metal pins were $1 * 0.25 \mathrm{~cm}$ ).

\section{Coactivation}

The CA paradigm used in the present study was the same as that described in a previous work about the effects of multifinger coactivation in young adults (Kalisch et al 2007). Five small solenoid devices (diameter of $8 \mathrm{~mm}$ each) were fixed to the tips of each finger of the right hand (ie, $\mathrm{d} 1=$ thumb, $\mathrm{d} 2=$ index finger, $\mathrm{d} 3=$ middle finger, $\mathrm{d} 4=$ ring finger, $\mathrm{d} 5=$ little finger) to transmit cutaneous stimuli to the dermal mechanoreceptors in the underlying skin portions. Laser vibrometer measurements revealed that the actual amplitude was approximately 100 microns. The CA sequence was played back via a portable digital storage and transmitted to a small amplifier that was connected to the solenoids. The sequence consisted of $10 \mathrm{~ms}$ pulses with a variable inter-stimulus interval of 100-3000 ms according to a Poisson distribution, resulting in an average stimulation frequency of $1 \mathrm{~Hz}$. During the three hours of application the compact devices allowed unrestricted mobility of the subjects in the target group. For subjects in the sham group the same set-up was used, however, no signal was transmitted from the digital storage to the amplifier. As the subjects were not informed about the characteristics of the CA, they were convinced to receive subthreshold stimulation.

\section{Experimental schedule and statistics}

All psychophysical experiments were carried out once prior to the multifinger CA (pre session) and three times after $\mathrm{CA}$, in order to evaluate changes in performance and a possible recovery of CA-induced effects (rec sessions). One session was conducted immediately after multifinger CA (post session), another one 24 hours later (rec-24 h), and the last one 4 days after multifinger CA (rec-96 $h$ ). As an exception the peg-board test was applied only in pre, post, 
and rec-96 $h$ session. All results are presented as means \pm standard deviations in the text.

To analyze group-specific changes in psychophysical performance we conducted repeated measures ANOVAs with factors GROUP and SESSION. Furthermore we conducted pairwise comparisons by calculating one-sided Dunnett posthoc tests (pre session as reference). A two-sided Dunnett test was chosen only for the analysis of data of the mislocalization test. Linear correlation analyses were calculated by means of two-sided Pearson-correlations (Spearman rank correlations respectively) and considered significant according to Bonferroni-corrected alpha-levels.

Additionally z-transformed false alarm rates (z(far)) and hit rates $(\mathrm{z}(\mathrm{hr}))$ were calculated for the subjects' two-point discrimination performance and used to obtain d-prime values (d'), as bias-free discrimination indices according to the formula " $d$ ' $=z(h r)-z(f a r)$ " (McNicol 1972; Wickens 2002). For the calculation of these indices we adjusted the false alarm rate to 0.125 by default, if no false alarm was detected in a given session.

\section{Results}

\section{Touch thresholds}

The touch thresholds of elderly subjects are not evenly distributed across the hand but decrease from the thumb to the little finger. In the pre condition we found no significant differences between touch thresholds of all fingers of the subjects in the target and the sham group (One-way ANOVA, factors GROUP*FINGER, $\left.\mathrm{F}_{(3,60)}=0.326, \mathrm{p}=0.860\right)$. The average threshold for $\mathrm{d} 1 \mathrm{was} 0.67 \pm 0.52 \mathrm{mN}, 0.36 \pm 0.22 \mathrm{mN}$ for $\mathrm{d} 2,0.25 \pm 0.10 \mathrm{mN}$ for $\mathrm{d} 3,0.22 \pm 0.05 \mathrm{mN}$ for $\mathrm{d} 4$, and $0.19 \pm 0.05 \mathrm{mN}$ for $\mathrm{d} 5$. Repeated measures ANOVA for factors GROUP and SESSION revealed no significant interactions for $\mathrm{d} 1, \mathrm{~d} 2, \mathrm{~d} 3$ and $\mathrm{d} 4\left(\mathrm{~F}_{(3,60)} \leq 2.611, \mathrm{p} \geq 0.060\right)$ but for $\mathrm{d} 5\left(\mathrm{~F}_{(3,60)}=2.831, \mathrm{p}=0.046\right)$ of the right hand.

The Dunnett post-hoc analyses revealed no significant threshold changes for the fingers $d 1-d 5$ after CA, neither for subjects in the target group $(\mathrm{p} \geq 0.698)$ nor for subjects in the sham group ( $p \geq 0.805$ ). These data demonstrate that in line with previous findings from adults, CA has no effect on touch threshold.

\section{Two-point discrimination thresholds}

Tactile acuity of the fingertips is not distributed equally across fingers, but is best at the thumb and index finger and declines across the remaining fingers (Weinstein 1968; Louis et al 1984; Desrosiers et al 1996; Kalisch et al 2007). For the pre condition, the discrimination thresholds assessed in the target and the sham group did not differ (One-way ANOVA, factors GROUP*FINGER, $\left.\mathrm{F}_{(3,60)}=0.371, \mathrm{p}=0.829\right)$. We found discrimination thresholds of $3.41 \pm 0.34 \mathrm{~mm}$ for $\mathrm{d} 1$, $3.52 \pm 0.35 \mathrm{~mm}$ for $\mathrm{d} 2,3.91 \pm 0.44 \mathrm{~mm}$ for $\mathrm{d} 3,4.32 \pm 0.48$ $\mathrm{mm}$ for $\mathrm{d} 4$, and $4.60 \pm 0.48 \mathrm{~mm}$ for $\mathrm{d} 5$. Spearman rank correlation analysis revealed a systematic relation between initial threshold (pre condition) and the position of the fingers (ie, d1-d5). Because this analysis was calculated on thresholds obtained before the intervention, data from both groups were used ( $n=110, r=0.744, p \leq 0.001)$.

After CA, discrimination thresholds were significantly lowered indicative of enhanced tactile acuity. Repeated measures ANOVA for factors GROUP and SESSION revealed no significant interaction for $\mathrm{d} 1$ of the right hand $\left(\mathrm{F}_{(3,60)}=2.009, \mathrm{p}=0.122\right)$ and left $\mathrm{d} 2\left(\mathrm{~F}_{(3,60)}=0.137\right.$, $\mathrm{p}=0.938)$, but for fingers $\mathrm{d} 2\left(\mathrm{~F}_{(3,60)}=6.134, \mathrm{p} \leq 0.001\right)$, $\mathrm{d} 3\left(\mathrm{~F}_{(3,60)}=3.767, \mathrm{p}=0.015\right), \mathrm{d} 4\left(\mathrm{~F}_{(3,60)}=6.598, \mathrm{p}=0.001\right)$, and $\mathrm{d} 5\left(\mathrm{~F}_{(3,60)}=6.844, \mathrm{p} \leq 0.001\right)$ of the right hand. The individual analysis of CA-evoked improvement of two-point discrimination performance in the target group by means of Dunnett post-hoc tests revealed a significant reduction of thresholds of $\mathrm{d} 1$ in the post session $(\mathrm{p}=0.007)$, of $\mathrm{d} 2$ in post $(\mathrm{p} \leq 0.001)$ and rec-24 $h$ session $(\mathrm{p} \leq 0.001)$ and of $\mathrm{d} 3, \mathrm{~d} 4$, $\mathrm{d} 5$ in post $(\mathrm{p} \leq 0.001)$, rec-24 $h(\mathrm{p} \leq 0.004)$, and rec-96 $h$ sessions $(\mathrm{p} \leq 0.010)$. Thresholds of the left index finger did not change between the sessions ( $\mathrm{p} \geq 0.317$ ) (Figure $2 \mathrm{a}$ ). Data obtained from subjects in the sham group did not indicate any significant changes neither on $\mathrm{d} 1(\mathrm{p} \geq 0.662)$, $\mathrm{d} 2$ ( $\mathrm{p} \geq 0.744), \mathrm{d} 3$ ( $\mathrm{p} \geq 0.460), \mathrm{d} 4(\mathrm{p} \geq 0.186)$, and $\mathrm{d} 5$ ( $p \geq 0.388)$, nor on the left $d 2$ ( $p \geq 0.572$ ) (Figure $2 b$ ).

The bias-free discrimination indices (d'-values) support the described differences between target group and sham group (Figure 2c). Repeated measures ANOVA for factors GROUP and SESSION revealed no significant interaction for $\mathrm{d} 1$ of the right hand $\left(\mathrm{F}_{(3,60)}=0.060, \mathrm{p}=0.981\right)$ and left $\mathrm{d} 2$ $\left(\mathrm{F}_{(3,60)}=0.387, \mathrm{p}=0.763\right)$, but for fingers $\mathrm{d} 2\left(\mathrm{~F}_{(3,60)}=7.519\right.$, $\mathrm{p} \leq 0.001), \mathrm{d} 3\left(\mathrm{~F}_{(3,60)}=3.957, \mathrm{p}=0.012\right), \mathrm{d} 4\left(\mathrm{~F}_{(3,60)}=5.820\right.$, $\mathrm{p}=0.001)$, and $\mathrm{d} 5\left(\mathrm{~F}_{(3,60)}=8.160, \mathrm{p} \leq 0.001\right)$ of the right hand. Individual analyses of discrimination indices of subjects in the target group by means of Dunnett post-hoc tests revealed a significant increase for d'-values of d1, d2, d3, d4, and $\mathrm{d} 5$ in post ( $\mathrm{p} \leq 0.001)$, rec-24 h session ( $\mathrm{p} \leq 0.004)$, and rec-96 $h$ session $(\mathrm{p} \leq 0.007)$. The averaged d'-values $(\mathrm{d} 1-\mathrm{d} 5$ right hand) of subjects in the target group increased from $1.14 \pm 0.18$ in pre session to $1.38 \pm 0.20$ in post session, $1.33 \pm 0.18$ in rec-24 $h$ session and $1.28 \pm 0.17$ in rec-96 $h$ session indicating improved acuity even 96 hours after the application of CA (Figure 2c). Averaged indices of the 

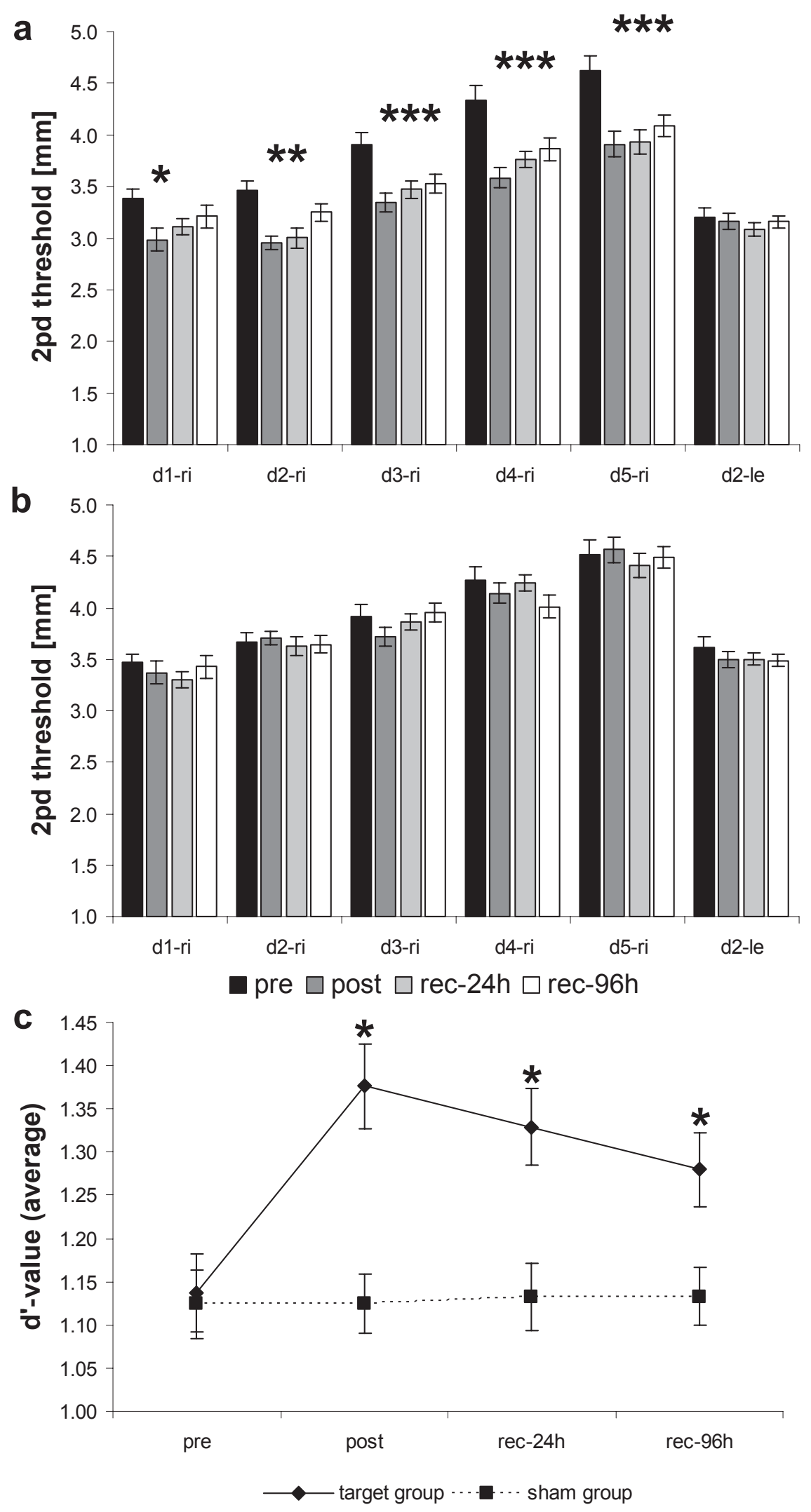

Figure 2 Spatial two-point discrimination thresholds were assessed on all fingers of the right hand (dI-ri-d5-ri) and the left index finger (d2-le) in all subjects of the target group $(n=16 ; a)$ and sham group $(n=6 ; \mathbf{b})$. After CA the thresholds of the right hand decreased significantly (stars indicate $p \leq 0.05)$ in the target group. Sham stimulation had no effect on the thresholds. The averaged bias-free discrimination indices (d'-values averaged across all fingers of the right hand) increased for subjects of the target group but not for subjects of the sham group in all measurements (post, rec- $24 \mathrm{~h}$, rec-96 h) after CA c). Error bars depict SEM. Stars indicate significant differences ( $\mathrm{P} \leq 0.05)$ to the pre condition. 
subjects in the sham group were $1.12 \pm 0.10$ in pre session, $1.12 \pm 0.09$ in post session, $1.13 \pm 0.10$ in rec-24 $h$ session and $1.13 \pm 0.08$ in rec-96 $h$ session (Figure $2 \mathrm{c}$ ).

\section{Mislocalization test}

In the pre condition, the distribution of mislocalizations of tactile stimuli did not differ between target group and sham group (One-way ANOVA, factors GROUP*NEIGHBOUR, $\left.\mathrm{F}_{(3,40)}=0.095, \mathrm{p}=0.962\right)$. When near-threshold tactile stimuli were applied to the fingers of the tested hand, $17.6 \pm 5.5 \%$ of all stimuli were mislocalized to a directly neighboring finger, $14.2 \pm 5.1 \%$ to a second neighbor, $4.2 \pm 3.6 \%$ to a third neighbor and $3.6 \pm 1.1 \%$ to a fourth neighbor. Repeated measures ANOVA for factors GROUP and SESSION revealed no significant interactions for number of mislocalizations on the first neighbor $\left(\mathrm{F}_{(3,42)}=1.945, \mathrm{p}=0.137\right)$, on the second neighbor $\left(\mathrm{F}_{(3,42)}=0.851, \mathrm{p}=0.477\right)$ and on the fourth neighbor $\left(\mathrm{F}_{(3,42)}=1.010, \mathrm{p}=0.451\right)$, but on the third neighbor $\left(\mathrm{F}_{(3,42)}=9.085, \mathrm{p}=0.001\right)$.

The comparison of the data of the target group by means of a two-tailed Dunnett post-hoc test showed a significant change of the mislocalization rate to the first neighboring finger from pre to post session (ie, a decrease from $17.5 \pm 5.0 \%$ to $10.3 \pm 1.9 \%, \mathrm{p}=0.002)$, but not to $\mathrm{rec}-24 \mathrm{~h}$ $(\mathrm{p}=0.338)$ and rec-96 $h$ session $(\mathrm{p}=0.633)$. The mislocalization rate to the second neighboring finger did not change between the sessions ( $p \geq 0.953$ ). However, the mislocalization rate to the third neighboring finger changed from pre to post session (ie, an increase from $4.8 \pm 3.7 \%$ to $15.8 \pm 2.9 \%$, $\mathrm{p} \leq 0.001$ ) and from pre to rec-24 $h$ session (ie, an increase from $4.8 \pm 3.7 \%$ to $10.0 \pm 1.4 \%, p=0.044)$. There was no significant difference between mislocalization rates of pre and rec-96 $h$ session $(\mathrm{p}=0.393)$. The mislocalization rates to the fourth neighboring finger did not change between the sessions ( $p \geq 0.221$ ) (Figure 3a).

Analyses of the data of the sham group revealed no changes between the sessions for mislocalization rates to the first neighboring finger ( $\mathrm{p} \geq 0.822$ ), the second neighbor ( $p \geq 0.403$ ), the third neighbor ( $\mathrm{p} \geq 0.730$ ), and forth neighbor ( $p \geq 0.709$ ) (Figure $3 b)$.

\section{Haptic object recognition}

The subjects' ability to identify objects by means of haptic exploration was quantified by the time to perform the task and the number of errors. There was no significant difference in time (One-way ANOVA, factor TIME, $\mathrm{F}_{(1,21)}=2.582$, $\mathrm{p}=0.124$ ) and number of errors (One-way ANOVA, factor ERROR, $\mathrm{F}_{(1,21)}=3.617, \mathrm{p}=0.072$ ) between target group and sham group in the pre session. The average time was $319.0 \pm 95.9 \mathrm{~s}$ and the average number of errors $4.7 \pm 2.5$. We hypothesized that the factors TIME and ERROR were inversely correlated, meaning that a high number of errors occurs when the test is performed quickly, and vice versa, however, the opposite was true. We found a positive correlation between both factors $(n=22, r=0.647, p \leq 0.001)$ (Figure $4 \mathrm{a}$ ) indicating that there is no trade-off between speed and accuracy.

Repeated measures ANOVA for factors GROUP and SESSION revealed no significant interactions for the time to complete the task $\left(\mathrm{F}_{(3,60)}=1.330, \mathrm{p}=0.273\right)$ and the number of errors $\left(\mathrm{F}_{(3,60)}=0.550, \mathrm{p}=0.650\right)$. Group-specific post-hoc analyses of both measures revealed changes in performance following the CA, but selectively in the target group. The time to perform the task was significantly reduced from $299.6 \pm$ $77.4 \mathrm{~s}$ in the pre session to $232.7 \pm 47.3 \mathrm{~s}$ in post session $(\mathrm{p}=0.004), 235.4 \pm 60.1 \mathrm{~s}$ in rec-24 $h$ session $(\mathrm{p}=0.006)$ and $232.8 \pm 57.2 \mathrm{~s}$ in rec-96 $h$ session $(\mathrm{p}=0.004$; Figure $4 \mathrm{~b})$. Number of errors decreased from $4.1 \pm 2.1$ in pre session to $2.4 \pm 1.6$ in post session $(\mathrm{p}=0.019), 2.7 \pm 1.9$ in rec $-24 h(\mathrm{p}$ $=0.051)$ and $2.8 \pm 2.1$ in rec-96 $h(\mathrm{p}=0.079$; Figure $4 \mathrm{c})$.

The time to perform the task was $370.7 \pm 127.3 \mathrm{~s}$ for subjects in the sham group for pre session and did not change in post session $(347.3 \pm 138.1 \mathrm{~s}, \mathrm{p}=0.635), 344.3 \pm 146.9 \mathrm{~s}$ in rec-24 $h$ session $(\mathrm{p}=0.620)$ and $333.5 \pm 146.3$ in rec-96 $h$ session $(p=0.561)$. There were also no changes for the number of errors. We measured $6.3 \pm 3.0$ in the pre session, $5.2 \pm 3.1$ in post session $(\mathrm{p}=0.530), 5.0 \pm 3.8$ in rec-24 $h$ session $(\mathrm{p}=0.480)$ and $4.6 \pm 3.8$ in rec-96 $h$ session $(\mathrm{p}=0.395)$.

\section{Peg-board test}

We quantified the subjects' ability to manipulate small objects by measuring the time to fulfill the peg-board test and the number of dropped items in a standard (long pins) and a demanding test form (short pins). The initial performance of the target group did not differ from the sham group, neither in the standard test form (One-way ANOVA, factor TIME, $\mathrm{F}_{(1,21)}=0.761, \mathrm{p}=0.393$; factor ERROR, $\mathrm{F}_{(1,21)}=1.229$, $\mathrm{p}=0.281$ ), nor in the demanding test form (One-way ANOVA, factor TIME, $\mathrm{F}_{(1,21)}=0.244, \mathrm{p}=0.627$; factor ERROR, $\left.\mathrm{F}_{(1,21)}=0.012, \mathrm{p}=0.914\right)$. The average time was $46.9 \pm 4.9 \mathrm{~s}$ and the average number of errors was $0.6 \pm 0.8$.

Repeated measures ANOVA for factors GROUP and SESSION revealed no significant interactions for the time to fulfill the standard peg-board test $\left(\mathrm{F}_{(2,40)}=1.528, \mathrm{p}=0.229\right)$ and the demanding test form $\left(\mathrm{F}_{(2,40)}=1.510, \mathrm{p}=0.223\right)$. The same analyses revealed no interaction for number of errors in 

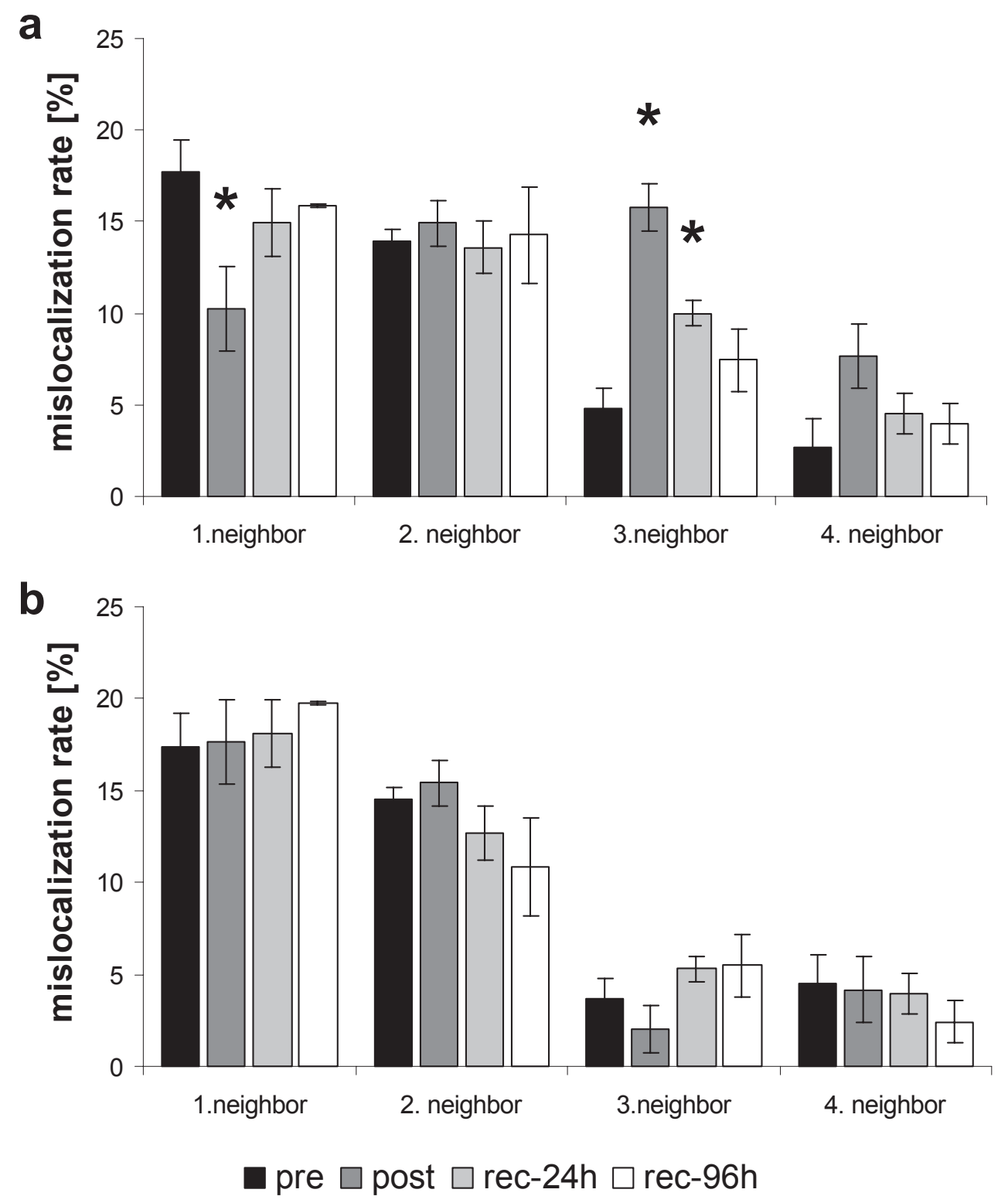

Figure 3 The rate of mislocalized tactile stimuli given by the number of stimulation in percent that were incorrectly perceived on fingers adjacent to the stimulated finger for subjects of the target group $(n=16$; $\mathbf{a})$ and the sham group $(n=16 ; \mathbf{b})$. We found that after CA in the target group, but not in subjects of the sham group the rate of mislocalized stimuli was shifted significantly from the directly neighboring to the third-neighboring finger. Error bars depict SEM. Stars indicate significant differences $(p \leq 0.05)$ to the pre condition.

the standard peg-board test $\left(\mathrm{F}_{(2,40)}=0.999, \mathrm{p}=0.377\right)$, but in the demanding test form $\left(\mathrm{F}_{(2,40)}=9.995, \mathrm{p} \leq 0.001\right)$.

The post-hoc analyses of data obtained from subjects of the target group revealed a significant reduction of the time to fulfill the peg-board test in both forms after CA. In the standard form the time decreased from $46.3 \pm 4.4 \mathrm{~s}$ in the pre session to $38.1 \pm 5.8 \mathrm{~s}$ in post $\operatorname{session}(\mathrm{p} \leq 0.001)$ and $41.5 \pm$ $7.2 \mathrm{~s}$ in rec-96 $h(\mathrm{p}=0.024)$. In the demanding form the time decreased from $62.2 \pm 13.8 \mathrm{~s}$ in pre session to $51.0 \pm 11.6 \mathrm{~s}$ in post $(\mathrm{p}=0.020)$ and $52.6 \pm 14.6 \mathrm{~s}$ in rec-96 $h(\mathrm{p}=0.043)$. Significant changes were also observed for the number of errors, which decreased from $0.8 \pm 0.9$ in the pre session of the standard test to $0.1 \pm 0.3$ in post $(\mathrm{p}=0.015)$ and $0.6 \pm$ 0.8 in rec-96 $h$ session $(\mathrm{p}=0.352)$. In the demanding test the number of errors decreased from $1.1 \pm 0.8$ in the pre session to $0.2 \pm 0.4$ in post $(\mathrm{p} \leq 0.001)$ and $0.3 \pm 0.6$ in rec-96 $h$ session $(\mathrm{p}=0.001)$. Data of subjects in the target group are displayed in Figures $5 \mathrm{a}$ and $5 \mathrm{~b}$ for the standard test and the demanding test form.

Data of the sham group on the other hand revealed no significant changes for the initial time to fulfill the test $(p \geq 0.262)$ and initial number of errors $(p \geq 0.667)$, 

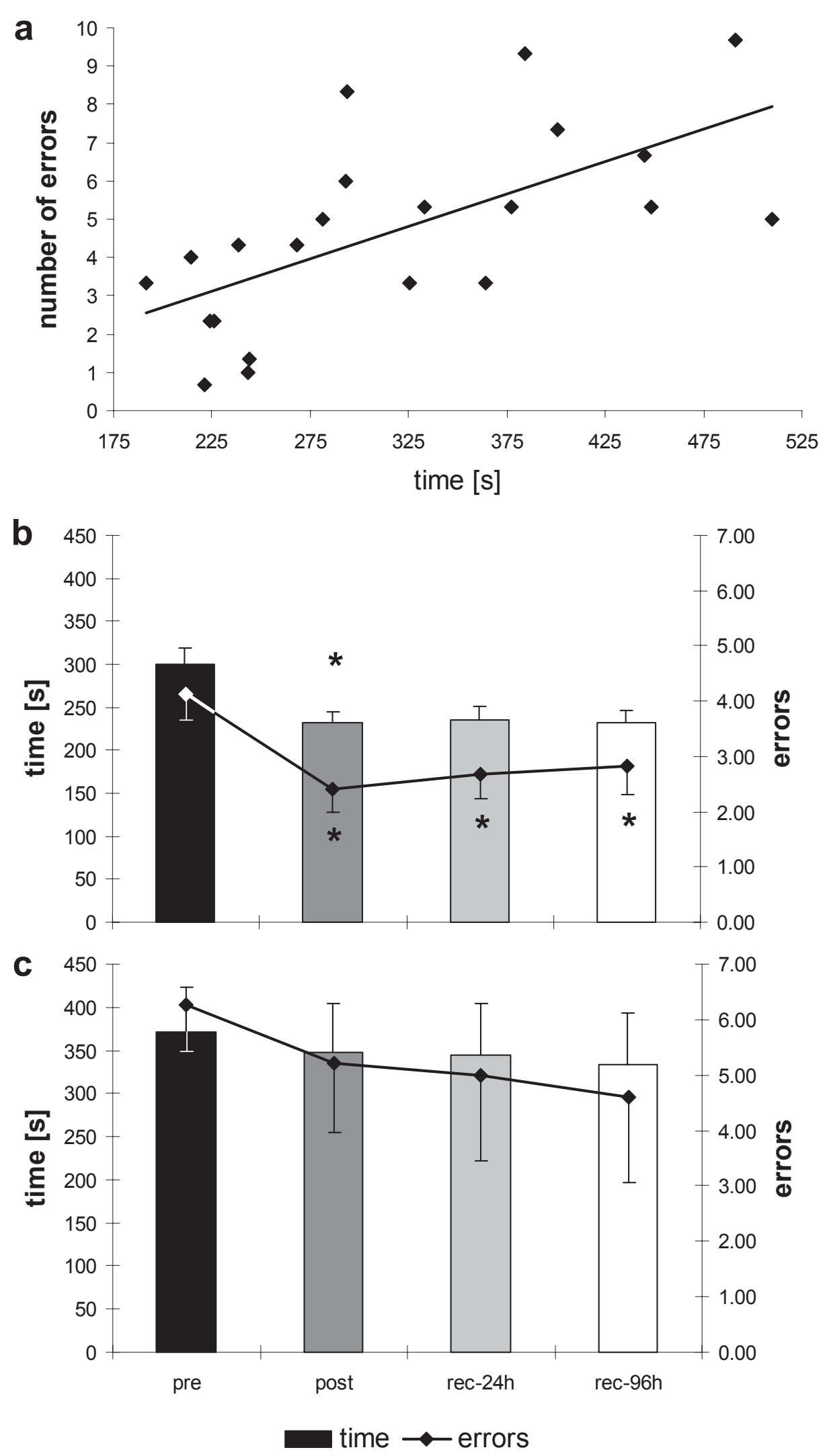

Figure 4 The subjects' haptic performance was assessed by recording the number of errors and the time to fulfill the object recognition test (see also Figure I). There was a significant correlation between number of errors and the time to fulfill the test for the pre session (Pearson, $n=22, r=0.647$, $p \leq 0.00$ I; (a). After CA, we found a significant decrease of the number of errors and the time to fulfill the test in the target group (b), but not in the data of the sham group (c). Error bars depict SEM. Stars indicate significant differences $(\mathrm{P} \leq 0.05)$ to the pre condition. 

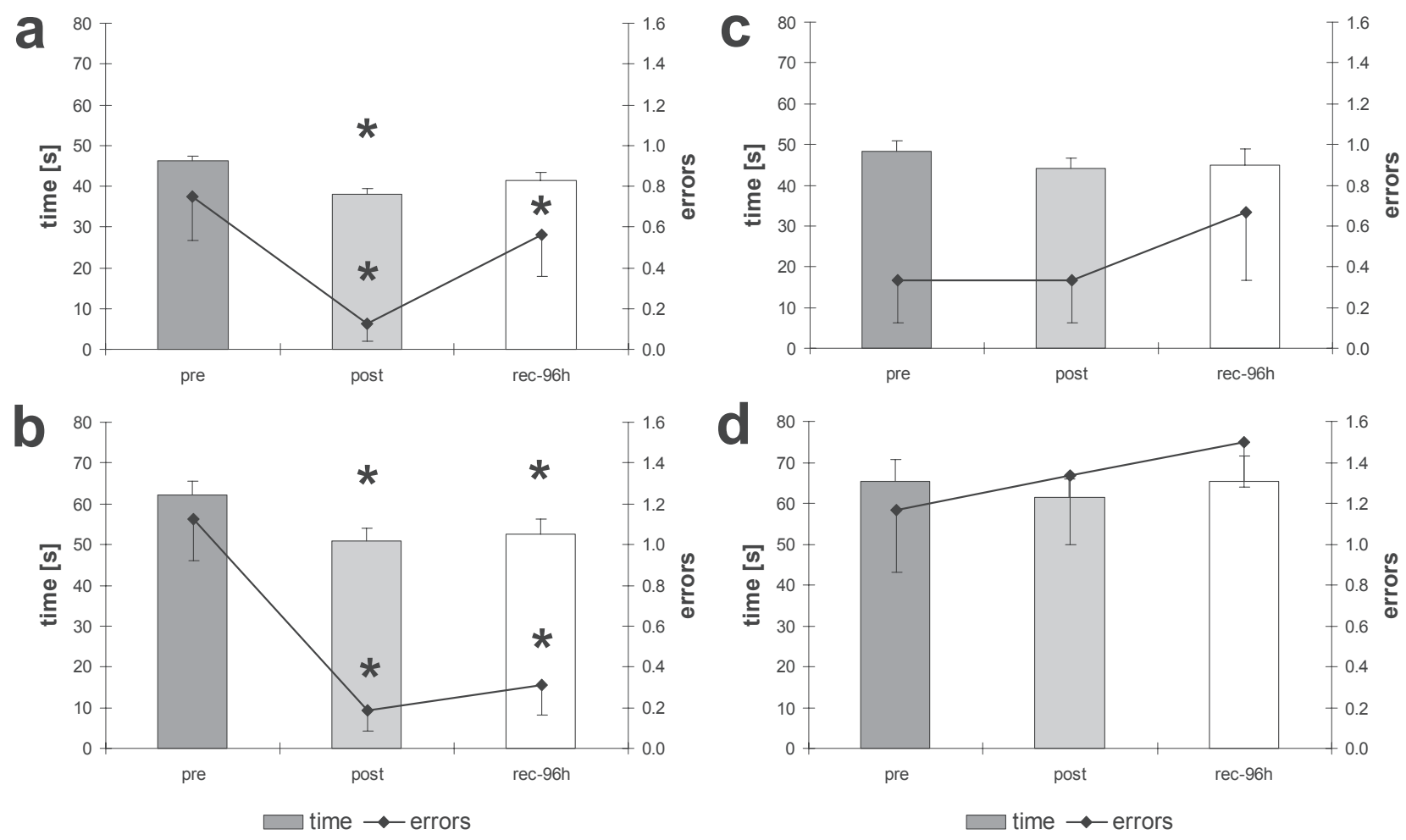

Figure $\mathbf{5}$ The peg-board test was used in a standard form using long pins (a:c) and in a demanding form using short pins (b:d).After CA, for subjects of the target group (a:b) we found a significant decrease of the number of errors (ie, number of dropped pins) and the time to fulfill the test, but not for the subjects in the sham group (c:d). Error bars depict SEM. Stars indicate significant differences $(p \leq 0.05)$ to the pre condition.

neither in the standard form $(48.4 \pm 6.3 \mathrm{~s}, 0.3 \pm 0.5$ errors; Figure $5 \mathrm{c})$ nor the demanding form $(66.4 \pm 12.7 \mathrm{~s}, 1.2 \pm 0.8$ errors; Figure 5d).

\section{Baseline-dependency of gains in performance}

In order to address the question whether CA effectiveness might be potentially dependent on the baseline performance of the subjects, we performed linear Pearson-correlation analyses between baseline (pre-condition) and post-condition for all tasks. For the two-point discrimination thresholds of subjects of the target group we found a significant correlation between their initial performance and the individual gain following CA: The correlation analysis revealed a stronger improvement for fingers with high initial thresholds and vice versa $(n=80, r=-0.587, p \leq 0.001)$ (Figure 6).

The same analysis was applied for the haptic object recognition performance of the subjects in the target group. We found a similar relation between initial performance and gain following $\mathrm{CA}$ for the factors time to fulfill the test $(\mathrm{n}=16, \mathrm{r}=-0.813, \mathrm{p} \leq 0.001)$ and number of errors $(\mathrm{n}=16, \mathrm{r}=-0.652, \mathrm{p}=0.006)$.
For the data obtained in the standard form of the pegboard test there was a significant correlation between the initial number of errors and the reduction in number of errors after $\mathrm{CA}(\mathrm{n}=16, \mathrm{r}=-0.921, \mathrm{p} \leq 0.001)$, but not for the time to fulfill the test and the accordant gain $(\mathrm{n}=16, \mathrm{r}=-0.100$, $p=0.713$ ). In contrast, for the demanding form of the pegboard test we found a correlation between initial performance and individual improvement after CA both for the time to fulfill the test $(n=16, r=-0.635, p=0.008)$ and the number of errors $(n=16, r=-0.866, p \leq 0.001)$.

In addition, we investigated potential correlations between the gains in performance in two-point discrimination, haptic object recognition and the peg-board test (Figure 7) by calculating linear Pearson correlations for the following factors: average gain (across $\mathrm{d} 1-\mathrm{d} 5$ ) in two-point discrimination performance, gains of time and errors in haptic object recognition, gains of time and errors in both forms of the pegboard test, whereas gain in performance refers to changes from pre to post session for the subjects of the target group. There was a complete lack of correlation between gains of two-point discrimination performance and gains in time and number of errors of the haptic object recognition 


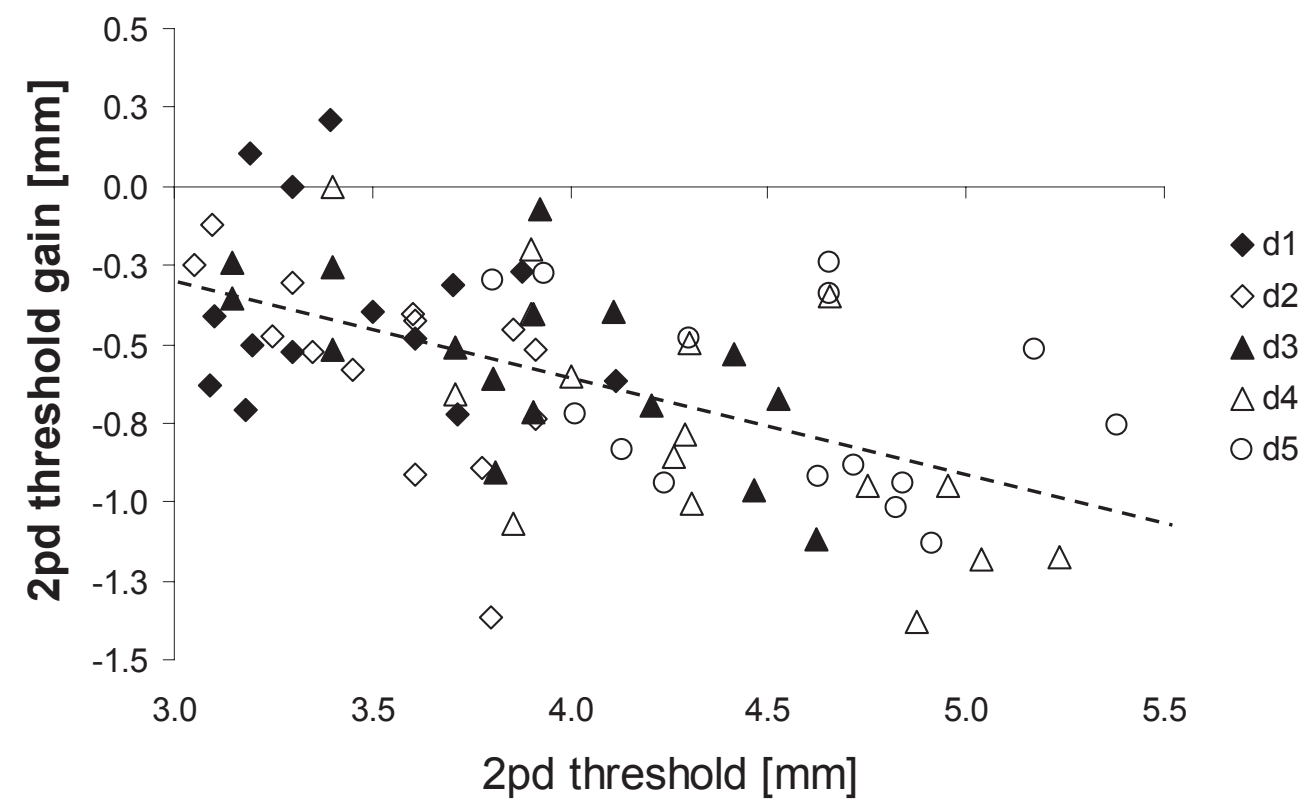

Figure 6 Linear correlation analysis (Pearson) between the two-point discrimination thresholds obtained on all fingers of the right hand in subjects of the target group $(n=16)$ and the threshold reduction from pre to post session (gain). The higher the initial threshold of a finger, the higher was the gain $(n=80, r=-0.587, p \leq 0.00 \mathrm{I})$.

test $(\mathrm{n}=16, \mathrm{r} \leq 0.194, \mathrm{p} \geq 0.472)$ and any form of the peg-board test $(\mathrm{n}=16, \mathrm{r} \leq 0.113, \mathrm{p} \geq 0.412)$.

Furthermore we found significant correlations between the gains in time and errors of the haptic object recognition task ( $\mathrm{n}=16, \mathrm{r}=0.598, \mathrm{p}=0.014)$, the number of errors of the haptic task and the standard version of the peg-board task $(n=16, r=0.500, p=0.490)$ and finally for the gain in time to fulfill the standard and the demanding version of the pegboard test $(\mathrm{n}=16, \mathrm{r}=0.601, \mathrm{p}=0.014)$.

\section{Discussion}

Here we demonstrate the effectiveness of a new intervention paradigm to improve human sensory-motor performance in old age. In the so-called CA, mechanical stimulation is

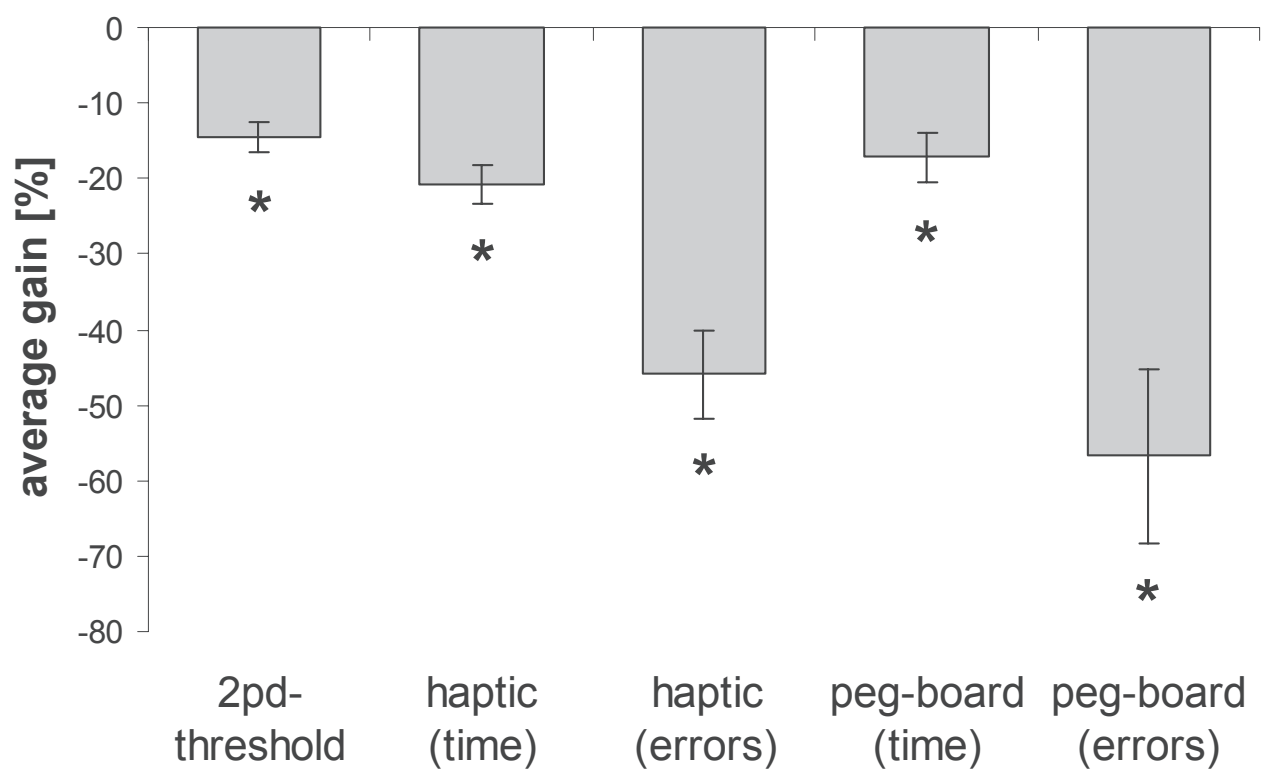

Figure 7 Summary of the tactile and sensorimotor improvement of subjects of the target group after CA. Gain in performance refers to percent changes from pre to post session. Two-point discrimination (2pd) thresholds were averaged for $\mathrm{dl}-\mathrm{d} 5$ of the right hand. For the peg-board test the time to fulfill the test and the number of errors are averages of the standard and demanding test form. Error bars depict SEM. Stars indicate significant differences $(p \leq 0.05)$ to the pre condition. 
applied simultaneously to the subjects' fingertips for three hours in order to drive plastic processes in cortical finger representations and to improve tactile performance (Pleger et al 2001, 2003; Dinse et al 2003; Kalisch et al 2007). We report a significant improvement of the subjects' two-point discrimination performance demonstrating increased tactile acuity following CA. Furthermore the subjects' ability for haptic object exploration and fine manipulative moments was improved as well. For all tasks that improved after CA, the individual gain was inversely related to the baseline performance, ie, subjects that were characterized by a poor baseline performance showed the largest improvement, while subjects with high baseline performance showed less benefit from CA (Dinse et al 2006).

From everyday experience we know that tactile sensibility is a crucial prerequisite for haptic performance (ie, identifying objects by exploratory hand or finger movements) and for fine-motor performance in general (Tremblay et al 2003; Lederman and Klatzky 2004). Even simple activities of everyday life like buttoning a shirt cannot be performed when sensory information from the fingertips is corrupted. In fact, we recently demonstrated the effects of mechanically shielding the fingertips by rubber coating on haptic and finemotor performance in young and elderly subjects (Dinse et al 2008). We found a stronger impairment in elderly subjects' performance as compared to young subjects after their tactile acuity was reduced by the coating, indicating an increasing need for availability of tactile information in old age.

Consequently, as tactile acuity is decreased significantly in old age (Thornbury and Mistretta 1981; Stevens 1992; Stevens and Patterson 1995; Stevens and Choo 1996; Stevens and Cruz 1996; Tremblay et al 2003), sensorimotor skills are negatively affected as well (Thornbury and Mistretta 1981; Cole 1991; Shiffman 1992; Cole and Beck 1994; Kinoshita and Francis 1996; Lazarus and Haynes 1997; Tremblay et al 2003). As a result of this development, everyday competence (ie, the ability to perform activities of daily living) is highly vulnerable in elderly people (Kinoshita and Francis 1996; Hughes et al 1997). In a first step to develop intervention measures to counteract these developments, we recently showed that tactile acuity can be improved through CA in elderly subjects demonstrating the effectiveness of the CA in subjects of old and very old age (Dinse et al 2006).

Here we provide a more systematic investigation that not only tested tactile acuity, but a wide range of tactile, haptic, and sensorimotor tasks that differ in their relation to motor behavior and in their cognitive demands.

\section{Touch threshold}

By comparing previously recorded touch thresholds in young subjects (Kalisch et al 2007) with data presented in the present work, we can confirm that touch thresholds are higher and more variable in elderly participants (Thornbury and Mistretta 1981). Furthermore we confirm again that the absolute touch thresholds, as measured by calibrated filaments, cannot be changed by CA (Kalisch et al 2007). In contrast, Collins and colleagues (1996) investigated the modifiability of touch thresholds in young and elderly subjects (Dhruv et al 2002) by means of electrical noise stimulation. They argued that stochastic resonance, which enhances the response of a nonlinear system to weak stimuli, acts to sensitize mechanoreceptors in the target skin area thereby making subthreshold stimuli detectable (Dhruv et al 2002). These findings suggest that stochastic resonance appears effective in interfering with human perception and behavior, however, its relation to $\mathrm{CA}$ remains unclear. In our view, stochastic resonance affects thresholds by enhancing inputs otherwise subthreshold, while CA alters the modes of cortical processing because of selective changes of synaptic efficacy and synaptic connections (Pleger et al 2003).

\section{Tactile acuity}

As repeatedly reported (Pleger et al 2001, 2003; Dinse et al 2003, 2005; Ragert et al 2004) the two-point discrimination threshold can be significantly improved by CA, even in elderly subjects (Dinse et al 2006). Here we demonstrate that CA can be extended to all fingers of the hand as recently described for young subjects (Kalisch et al 2007). Similar to young subjects we found an improvement of tactile acuity as documented by a lowering of discrimination thresholds and an increase of the bias-free discrimination indices (d'-values). Furthermore, the use of multifinger CA had the effect to stabilize the $\mathrm{CA}$-induced improvement. While under conditions of single-finger CA the improvement recovered to baseline after about 24 hours (Dinse et al 2006), we found that the improvement was preserved even after 96 hours after CA. The longer-lasting effects seem to be specific for elderly subjects, as the improvement evoked in young subjects by the identical multifinger CA protocol was limited to 24 hours (Kalisch et al 2007).

While it is undisputed that the density of mechanoreceptors in the skin decreases with age (Dorfman and Bosley 1979; Bruce 1980) there is a controversial discussion about the role of receptor density in tactile acuity. While the density of Meissner's corpuscles in the index and ring fingers does not differ, the acuity does. On the other hand, 
while males have lower density of Meissner's corpuscles compared with females, their acuity is not different (Dillon et al 2001). Furthermore, fingertip skin conformance was shown to account for some differences in tactile acuity in young subjects, but not for the decline in spatial acuity with aging (Vega-Bermudez and Johnson 2004). Most importantly, recent findings demonstrated that the typical agerelated decline in tactile performance is not inevitable, but that performance can be recovered by learning and training (Dinse et al 2006), despite the accumulation of degenerative processes during aging. These results indicate that mechanoreceptor density may play a minor role in determining tactile acuity performance at old age. We therefore conclude that the observed lowering of thresholds after CA is most presumably mediated by cortical mechanisms. This view is also supported by recent imaging data demonstrating a correlation between tactile hyperacuity and cortical map size of the fingers in the primary somatosensory cortex (Duncan and Boynton, 2007).

\section{Mislocalization}

While discrimination performance requires the differentiation between two stimuli, localization describes the ability to identify the correct position of one stimulus. In the finger localization tests subjects are asked to report which finger of a hand had been stimulated. Here we report that under conditions of synchronous multifinger CA finger localization performance is impaired in elderly subjects. A similar result has recently been described for young adults (Kalisch et al 2007). Accordingly, CA exerts not exclusively beneficial effects, but can lead to impaired performance in a task-dependent way.

In case of the finger localization test, CA leads to an increase in localization errors. Closer analysis revealed that these changes consisted of a decrease of localization errors on directly neighboring fingers, while on more distant fingers the number of mislocalizations increased. At the cortical level, single finger CA is known to result in an expansion of the finger representation being stimulated (Pleger et al 2003). Provided that multifinger CA as employed in this study evokes comparable changes, substantial overlap can be expected to occur between finger representations. As a consequence, correct localization performance between more distant fingers drops. This assumption is supported by a study from Pilz and colleagues (2004), who investigated the effects of CA on human cortical finger representations by means of functional imaging. They found that synchronous CA leads to an overlap of finger representations in somatosensory cortex and thereby caused an increase in the frequency of mislocalizations between the stimulated fingers. On the contrary, asynchronous stimulation evoked a segregation of the corresponding finger representations (Pilz et al 2004).

The relation between mislocalization of tactile stimuli and cortical organization in the human somatosensory system has been extensively studied in blind subjects. Sterr and colleagues (1998a, 1998b, 2003) showed that multifinger Braille readers with high tactile acuity showed increased frequency of mislocalization between the fingers used for Braille reading as compared to normal, sighted people, suggesting increased mislocalization was related to Braille reading skills rather than blindness in general. Interestingly, based on magnetoencephalography data, blind subjects were characterized by a disturbed topography of the finger representations (Sterr et al 1998a, 1998b). It was therefore concluded that the increase of localization errors was due to disarranged cortical maps.

More generally, there seems to be a trade-off between discrimination and localization behavior, which can be observed under training conditions as well as under conditions of passive stimulation. The latter indicates that CA evokes tactile learning processes similar to those induced by training and everyday-life situations. These findings imply that cortical processing modes exist which cannot be optimized in parallel. In this view, the average performance encountered in an individual will most likely reflect a balanced compromise in trying to achieve optimal, but not maximal performance. Through learning and plasticity processes it is possible to further enhance certain skills, but not all in parallel, resulting in an improvement of some skills at the expense of impairment of others.

Tommerdahl and colleagues (2007) recently investigated the impact of mechanical skin stimulation on adult subjects' ability for sensory information processing, ie, temporal order judgement (TOJ) and temporal discriminative thresholds (TDT). They found that in the presence of a $25 \mathrm{~Hz}$ conditioning mechanical stimulus that was delivered before, concurrently and after the task on fingers $\mathrm{d} 2$ and $\mathrm{d} 3$, TOJ was significantly impaired, which was not the case when the mechanical stimulation was either applied bilaterally or in asynchronous manner. These results strongly support the theory that the synchronization of cortical ensembles in somatosensory cortex can change sensory perception (Tommerdahl et al 2007).

\section{Haptic performance}

Besides the CA-induced improvement of tactile acuity we addressed the modifiability of haptic performance by CA. We demonstrate that in elderly subjects application of CA 
not only improves tactile acuity, but also haptic performance. According to the data from the sham group, performance improves slightly if the test is repeatedly executed; this effect, however, is small compared to the CA-evoked effect. Because haptic exploration requires a certain level of acuity, one might expect a strong relation between individual measures of acuity and haptic performance. The lack of correlation between individual changes in tactile and haptic performance might be explained by the cross-modal nature of the haptic test, ie, haptic exploration but visual comparison to the given samples. Furthermore the test includes cognitive components such as the explorative process of unfamiliar objects which requires visual imagery. We therefore assume that these additional requirements make haptic performance a multi-factorial process, in which the individual contribution of tactile acuity cannot readily be identified. Cognitive components in haptic performance of elderly subjects are subject of a recent study of our group (unpublished data).

\section{Fine motor performance}

Age-related changes in fine-motor performance are welldocumented (Thornbury and Mistretta 1981; Cole 1991; Shiffman 1992; Cole and Beck 1994; Kinoshita and Francis 1996; Lazarus and Haynes 1997). By using a peg-board test we studied whether CA affects motor performance. Although so far CA has only been shown to improve tactile perception, our data demonstrates that CA can as well improve fine motor performance in elderly subjects.

Local anesthesia studies demonstrated that the suppression of cutaneous inputs affects complex motor tests more than simple tests (Ebied 2003). This result may explain why the use of a challenging peg-board test instead of simple grasp and lift tests enabled us to detect changes in performance after CA. Furthermore, we found a stronger improvement after $\mathrm{CA}$ for the demanding version of the peg-board test as compared to the standard version.

The observed CA-induced improvement of fine-motor performance could have been caused by enhanced spatial discrimination. In this view, superior tactile acuity improves the ability to apply an accurate precision grip, because the position of the manipulated object can be perceived in more detail and the detection of slip events might be improved (Witney et al 2004). However, in the present study we observed a lack of correlation between improvement of tactile acuity and fine motor performance. We know from clinical experiments with patients that sensory stimulation not only affects the subsequent sensory representations, but also alters motor performance directly and with long-lasting effects by mechanisms of cross-system plasticity (Hamdy et al 1998; Wu et al 2006). These data corroborated earlier cortical plasticity experiments done in animals, which indicated that temporary changes in sensory input can produce persistent changes in the organization of sensory (Jenkins et al 1990; Wang et al 1995) and motor (Nudo et al 1996) areas of the cerebral cortex. Whatever the underlying mechanisms of cortical plasticity are, our data support the impact of sensory stimulation for the restoration of motor performance in elderly.

\section{Baseline dependency}

On average, CA induced an overall gain of performance in all tasks tested except for touch thresholds, which were, however, highly variable from subject to subject. Closer analysis revealed that the amount of coactivation-induced changes depended on the baseline performance of the subjects, ie, the initial performance of a subject determines the level of improvement. A similar observation was reported for chronic stroke patients. Application of a single two hour session of somatosensory stimulation on the paretic hand elicited a significant improvement in hand functions. The magnitude of this improvement was more prominent in patients with stronger impairment, supporting the notion of a baseline dependency in somatosensory interventional paradigms (Wu et al 2006).

Baseline dependancy is a phenomenon often occurring in perceptual learning. For example, in learning of vernier discrimination and depth perception in visual perceptual learning a substantial amount of interobserver variability can be accounted for by the initial level of performance (Fahle and Henke-Fahle 1996). The authors reported that in general improvement through learning was most pronounced in observers who yielded the highest thresholds initially and vice versa. While we found a clear baseline dependence for coactivation-induced changes of two-point discrimination in elderly, no such dependency had been observed previously in coactivation-induced improvement in spatial discrimination in young subjects (Dinse et al 2003). The nature of such baseline dependency remains largely elusive. Conceivably, ceiling effects may cause baseline-dependencies as found here, but the findings from long-term training suggest that subjects can improve quite substantially beyond the gain found in our studies (Ragert et al 2004).

\section{Conclusion}

The present study demonstrates the impact of sensory CA on improving sensorimotor performance in the elderly. Besides a significant improvement of tactile acuity in elderly people, we report for the first time that $\mathrm{CA}$ improves haptic object 
exploration skills and fine motor object manipulation abilities. The preservation of sufficient tactile and haptic performance into high age as shown after $\mathrm{CA}$ is an important prerequisite for maintaining an independent and autonomous lifestyle. We therefore believe that the concept of CA can turn out beneficial in preserving everyday sensorimotor competence. Taken together, the documented effects of CA make it a likely candidate for future interventions in elderly and maybe also in patients with somatosensory dysfunctions.

\section{Author contributions}

TK conceived of the study, carried out the psychophysical measurements, performed the statistical analyses, and drafted the manuscript. MT participated in the study design and helped to draft the manuscript. HRD participated in the study design and coordination and helped to draft the manuscript. All authors read and approved the final manuscript.

\section{Acknowledgments}

We are grateful to M. Ziesmer for excellent technical support and Dr. S. Lissek for proofreading the manuscript. This work was supported by grants from the DFG to HRD (Di 334/10-4) and MT (Te 315/2-1) and a grant from the Schering Stiftung was awarded to TK.

\section{References}

Bell-Krotoski JA, Fess EE, Figarola JH, et al. 1995. Threshold detection and Semmes-Weinstein monofilaments. J Hand Ther, 8:155-62.

Besne I, Descombes C, Breton, L. 2002. Effect of age and anatomical site on density of sensory innervation in human epidermis. Arch Dermatol, 138:1445-50.

Birznieks I, Jenmalm P, Goodwin AW, et al. 2001. Encoding of direction of fingertip forces by human tactile afferents. J Neurosci, 21:822-837.

Bolton CF, Winkelmann RK, Dyck PJ. 1966. A quantitative study of Meissner's corpuscles in man. Neurology, 16:1-9.

Bouche P, Cattelin F, Saint-Jean O, et al. 1993. Clinical and electrophysiological study of the peripheral nervous system in the elderly. J Neurol, 240:263-8.

Braun C, Ladda J, Burkhardt M, et al. 2005. Objective measurement of tactile mislocalization. IEEE Trans Biomed Eng, 52:728-35.

Bruce MF. 1980. The relation of tactile thresholds to histology in the fingers of elderly people. J Neurol Neurosurg Psychiatry, 43:730-4.

Buonomano DV, Merzenich MM. 1998. Cortical plasticity: from synapses to maps. Аnпu Rev Neurosci, 21:149-86.

Cabeza R, Anderson ND, Locantore JK, et al. 2002. Aging gracefully: compensatory brain activity in high-performing older adults. Neuroimage, 17:1394-402.

Caruso G, Nilsson J, Crisci C, et al. 1993. Sensory nerve findings by tactile stimulation of median and ulnar nerves in healthy subjects of different ages. Electroencephalogr Clin Neurophysiol, $89: 392-8$

Cauna N. 1987. The effects of aging on the receptor organs of the human dermis. In: Montagna W (Ed.). Advances in the Biology of the Skin. New York: Pergamon.

Chodzko-Zajko WJ. 1997. Normal aging and human physiology. Semin Speech Lang, 18:95-104; quiz 104-5.
Cole KJ. 1991. Grasp force control in older adults. J Mot Behav, 23:251-8.

Cole KJ, Beck CL. 1994. The stability of precision grip force in older adults. J Mot Behav, 26:171-7.

Collins JJ, Imhoff TT, Grigg P. 1996. Noise-enhanced tactile sensation. Nature, 383:770.

Craig JC, Rhodes RP. 1992. Measuring the error of localization. Behav Res Meth Instrum Comput, 24:511-14.

Cua AB, Wilhelm KP, Maibach HI. 1990. Elastic properties of human skin: relation to age, sex, and anatomical region. Arch Dermatol Res, 282: $283-8$.

Desrosiers J, Hebert R, Bravo G, et al. 1996. Hand sensibility of healthy older people. J Am Geriatr Soc, 44:974-8.

Dhruv NT, Niemi JB, Harry JD, et al. 2002. Enhancing tactile sensation in older adults with electrical noise stimulation. Neuroreport, 13:597-600.

Dillon YK, Haynes J, Henneberg M. 2001. The relationship of the number of Meissner's corpuscles to dermatoglyphic characters and finger size. J Anat, 199:577-84.

Dinse H, Kalisch T, Ragert P, et al. 2005. Improving human haptic performance in normal and impaired human populations through unattended activation-based learning. ACM Trans Appl Percept, 2:71-88.

Dinse H, Wilimzig C, Kalisch T. 2008. Learning in haptic perception. In: Grunwald M(Ed.). Human Haptic Perception - Basics and Applications. Basel, Switzerland: Birkhäuser.

Dinse HR, Kleibel N, Kalisch T, et al. 2006. Tactile coactivation resets age-related decline of human tactile discrimination. Ann Neurol, 60:88-94.

Dinse HR, Merzenich MM. 2002. Adaptation of Inputs in Somatosensory System, Boston: MIT Press.

Dinse HR, Ragert P, Pleger B, et al. 2003. Pharmacological modulation of perceptual learning and associated cortical reorganization. Science, 301:91-4.

Dorfman LJ, Bosley TM. 1979. Age-related changes in peripheral and central nerve conduction in man. Neurology, 29:38-44.

Duncan RO, Boynton GM. 2007. Tactile hyperacuity thresholds correlate with finger maps in primary somatosensory cortex (S1). Cereb Cortex, 17:2878-91.

Ebied AM. 2003. The role of cutaneous sensation in the motor function of the hand. J Orthop Res, 22:862-6.

Elbert T, Pantev C, Wienbruch C, et al. 1995a. Increased cortical representation of the fingers of the left hand in string players. Science, 270:305-7.

Elbert T, Pantev C, Wienbruch C, et al. 1995b. Increased use of the left hand in string players associated with increased cortical representation of the fingers. Science, 220:21-3.

Evarts EV, Fromm C. 1979. Sensory responses in motor cortex neurons during precise motor control. Neurosci Lett, 5:267-72.

Fahle M, Henke-Fahle S. 1996. Interobserver variance in perceptual performance and learning. Invest Ophthalmol Vis Sci, 37:869-77.

Falconer J, Hughes SL, Naughton BJ, et al. 1991. Self report and performance-based hand function tests as correlates of dependency in the elderly. J Am Geriatr Soc, 39:695-9.

Folstein MF, Folstein SE, McHugh PR. 1975. "Mini-mental state". A practical method for grading the cognitive state of patients for the clinician. J Psychiatr Res, 12:189-98.

Giampaoli S, Ferrucci L, Cecchi F, et al. 1999. Hand-grip strength predicts incident disability in non-disabled older men. Age Ageing, 28:283-8.

Godde B, Stauffenberg B, Spengler F, et al. 2000 Tactile coactivationinduced changes in spatial discrimination performance. $J$ Neurosci, 20:1597-604.

Goodwin AW, Wheat HE. 2004. Sensory signals in neural populations underlying tactile perception and manipulation. Annu Rev Neurosci, 27:53-77.

Gysin P, Kaminski TR, Gordon AM. 2003. Coordination of fingertip forces in object transport during locomotion. Exp Brain Res, 149:371-9. 
Hamdy S, Rothwell JC, Aziz Q, et al. 1998. Long-term reorganization of human motor cortex driven by short-term sensory stimulation. Nat Neurosci, 1:64-8.

Hashimoto I, Suzuki A, Kimura T, et al. 2004. Is there training-dependent reorganization of digit representations in area $3 \mathrm{~b}$ of string players? Clin Neurophysiol, 115:435-47.

Hughes S, Gibbs J, Dunlop D, et al. 1997. Predictors of decline in manual performance in older adults. J Am Geriatr Soc, 45:905-10.

Iwasaki T, Goto N, Goto J, et al. 2003. The aging of human Meissner's corpuscles as evidenced by parallel sectioning. Okajimas Folia Anat Jpn, 79:185-9.

Jeannerod M, Arbib MA, Rizzolatti G, et al. 1995. Grasping objects: the cortical mechanisms of visuomotor transformation. Trends Neurosci, 18:314-20.

Jenkins WM, Merzenich MM, Ochs MT, et al. 1990. Functional reorganization of primary somatosensory cortex in adult owl monkeys after behaviorally controlled tactile stimulation. J Neurophysiol, 63:82-104.

Johansson RS, Westling G. 1984. Roles of glabrous skin receptors and sensorimotor memory in automatic control of precision grip when lifting rougher or more slippery objects. Exp Brain Res, 56:550-64.

Johansson RS, Westling G. 1987. Significance of cutaneous input for precise hand movements. Electroencephalogr Clin Neurophysiol Suppl, 39:53-7.

Kalisch T, Tegenthoff M, Dinse HR. 2007. Differential effects of synchronous and asynchronous multifinger coactivation on human tactile performance. BMC Neurosci, 8:58.

Kinoshita H, Francis PR. 1996. A comparison of prehension force control in young and elderly individuals. Eur J Appl Physiol Occup Physiol, 74:450-60.

Klatzky RL, Lederman SJ. 1995. Identifying objects from a haptic glance. Percept Psychophys, 57:1111-23.

Klatzky RL, Lederman SJ, Metzger VA. 1985. Identifying objects by touch: an "expert system". Percept Psychophys, 37:299-302.

Krampe RT. 2002. Aging, expertise and fine motor movement. Neurosci Biobehav Rev, 26:769-76.

Lazarus JA, Haynes JM. 1997. Isometric pinch force control and learning in older adults. Exp Aging Res, 23:179-99.

Lederman SJ, Klatzky RL. 1993. Extracting object properties through haptic exploration. Acta Psychol (Amst), 84:29-40.

Lederman SJ, Klatzky RL. 2004. Haptic identification of common objects effects of constraining the manual exploration process. Percept Psychophys, 66:618-28.

Liepert J, Tegenthoff M, Malin JP. 1995. Changes of cortical motor area size during immobilization. Electroencephalogr Clin Neurophysiol, 97:382-6.

Louis DS, Greene TL, Jacobson KE, et al. 1984. Evaluation of normal values for stationary and moving two-point discrimination in the hand. $J$ Hand Surg [Am], 9:552-5.

McNicol D. 1972. A Primer in Signal Detection Theory. London: Allen and Unwin.

Moberg E. 1983. The role of cutaneous afferents in position sense, kinaesthesia, and motor function of the hand. Brain, 106:1-19.

Monzee J, Lamarre Y, Smith AM. 2003. The effects of digital anesthesia on force control using a precision grip. J Neurophysiol, 89:672-83.

Nudo RJ, Milliken GW, Jenkins WM, et al. 1996. Use-dependent alterations of movement representations in primary motor cortex of adult squirrel monkeys. J Neurosci, 16:785-807.

Oldfield RC. 1971. The assessment and analysis of handedness: the Edinburgh inventory. Neuropsychologia, 9:97-113.

Pantev C, Oostenveld R, Engelien A, et al. 1998. Increased auditory cortical representation in musicians. Nature, 392:811-14.

Pascual-Leone A, Torres F. 1993. Plasticity of the sensorimotor cortex representation of the reading finger in Braille readers. Brain, 116(Pt 1):39-52.

Pilz K, Veit R, Braun C, et al. 2004. Effects of co-activation on cortical organization and discrimination performance. Neuroreport, 15:2669-72.
Pleger B, Dinse HR, Ragert P, et al. 2001. Shifts in cortical representations predict human discrimination improvement. Proc Natl Acad Sci USA, 98:12255-60.

Pleger B, Foerster AF, Ragert P, et al. 2003. Functional imaging of perceptual learning in human primary and secondary somatosensory cortex. Neuron, 40:643-53.

Quilliam TA, Ridley A. 1971. The receptor community in the finger tip. J Physiol, 216:15P-17P.

Ragert P, Kalisch T, Bliem B, et al. 2008. Differential effects of tactile high- and low-frequency stimulation on tactile discrimination in human subjects. BMC Neurosci, 9:9.

Ragert P, Schmidt A, Altenmuller E, et al. 2004. Superior tactile performance and learning in professional pianists: evidence for meta-plasticity in musicians. Eur J Neurosci, 19:473-8.

Raz N, Gunning FM, Head D, et al. 1997. Selective aging of the human cerebral cortex observed in vivo: differential vulnerability of the prefrontal gray matter. Cereb Cortex, 7:268-82.

Recanzone GH, Jenkins WM, Grajski KA, et al. 1992. Topographic reorganization of the hand representation in cortical area $3 \mathrm{~b}$ owl monkeys trained in a frequency-discrimination task. J Neurophysiol, 67:1031-56

Recanzone GH, Schreiner CE, Merzenich MM. 1993. Plasticity in the frequency representation of primary auditory cortex following discrimination training in adult owl monkeys. $J$ Neurosci, 13:87-103.

Reding MJ, Potes E. 1988. Rehabilitation outcome following initial unilateral hemispheric stroke. Life table analysis approach. Stroke, 19:1354-8.

Rivner MH, Swift TR, Malik K. 2001. Influence of age and height on nerve conduction. Muscle Nerve, 24:1134-41.

Sathian K, Zangaladze A, Green J, et al. 1997. Tactile spatial acuity and roughness discrimination: impairments due to aging and Parkinson's disease. Neurology, 49:168-77.

Schweizer R, Braun C, Fromm C, et al. 2001. The distribution of mislocalizations across fingers demonstrates training-induced neuroplastic changes in somatosensory cortex. Exp Brain Res, 139:435-42.

Schweizer R, Maier M, Braun C, et al. 2000. Distribution of mislocalizations of tactile stimuli on the fingers of the human hand. Somatosens Mot Res, 17:309-16.

Shiffman LM. 1992. Effects of aging on adult hand function. Am J Occup Ther, 46:785-92.

Smania N, Montagnana B, Faccioli S, et al. 2003. Rehabilitation of somatic sensation and related deficit of motor control in patients with pure sensory stroke. Arch Phys Med Rehabil, 84:1692-702.

Sowell ER, Peterson BS, Thompson PM, et al. 2003. Mapping cortical change across the human life span. Nat Neurosci, 6:309-15.

Sterr A, Green L, Elbert T. 2003. Blind Braille readers mislocate tactile stimuli. Biol Psychol, 63:117-27.

Sterr A, Muller MM, Elbert T, et al. 1998a. Changed perceptions in Braille readers. Nature, 391:134-5.

Sterr A, Muller MM, Elbert T, et al. 1998b. Perceptual correlates of changes in cortical representation of fingers in blind multifinger Braille readers. J Neurosci, 18:4417-23.

Stevens JC. 1992. Aging and spatial acuity of touch. J Gerontol, 47:P35-40.

Stevens JC, Choo KK. 1996. Spatial acuity of the body surface over the life span. Somatosens Mot Res, 13:153-66.

Stevens JC, Cruz LA. 1996. Spatial acuity of touch: ubiquitous decline with aging revealed by repeated threshold testing. Somatosens Mot Res, 13:1-10.

Stevens JC, Patterson MQ. 1995. Dimensions of spatial acuity in the touch sense: changes over the life span. Somatosens Mot Res, 12:29-47.

Tamburin S, Manganotti P, Zanette G, et al. 2004. Cutaneomotor integration in human hand motor areas: somatotopic effect and interaction of efferents. Exp Brain Res, 141:232-41.

Tecchio F, Padua L, Aprile I, et al. 2002. Carpal tunnel syndrome modifies sensory hand cortical somatotopy: a MEG study. Hum Brain Mapp, 17:28-36. 
Tegenthoff M, Ragert P, Pleger B, et al. 2005. Improvement of tactile discrimination performance and enlargement of cortical somatosensory maps after $5 \mathrm{~Hz}$ rTMS. PLoS Biol, 3:e362.

Thornbury JM, Mistretta CM. 1981. Tactile sensitivity as a function of age. J Gerontol, 36:34-9.

Tommerdahl M, Tannan V, Zachek M, et al. 2007. Effects of stimulus-driven synchronization on sensory perception. Behav Brain Funct, 3:61.

Tremblay F, Wong K, Sanderson R, et al. 2003. Tactile spatial acuity in elderly persons: assessment with grating domes and relationship with manual dexterity. Somatosens Mot Res, 20:127-32.

Valerio BC, Nobrega JA, Tilbery CP. 2004. Neural conduction in hand nerves and the physiological factor of age. Arq Neuropsiquiatr, 62:114-18.

Vega-Bermudez F, Johnson KO. 2004. Fingertip skin conformance accounts, in part, for differences in tactile spatial acuity in young subjects, but not for the decline in spatial acuity with aging. Percept Psychophys, 66:60-7.

Verbrugge LM, Jette AM. 1994. The disablement process. Soc Sci Med, $38: 1-14$.

Wang X, Merzenich MM, Sameshima K, et al. 1995. Remodelling of hand representation in adult cortex determined by timing of tactile stimulation. Nature, 378:71-5.
Weinstein S. 1968. Intensive and extensive aspects of tactile sensitivity as a function of body part, sex, and laterality. In: Kenshalo D (Ed.). The Skin Senses. Springfield: Charles C Thomas.

Wickens TD. 2002. Elementary Signal Detection Theory. Oxford: Oxford University Press.

Williams ME, Hadler NM, Earp JA. 1982. Manual ability as a marker of dependency in geriatric women. J Chronic Dis, 35:115-22.

Witney AG, Wing A, Thonnard JL, et al. 2004. The cutaneous contribution to adaptive precision grip. Trends Neurosci, 27:637-43.

Woodward KL. 1993. The relationship between skin compliance, age, gender, and tactile discriminative thresholds in humans. Somatosens Mot Res, 10:63-7.

Wu CW, Seo HJ, Cohen LG. 2006. Influence of electric somatosensory stimulation on paretic-hand function in chronic stroke. Arch Phys Med Rehabil, 87:351-7.

Young A. 1997. Ageing and physiological functions. Philos Trans R Soc Lond B Biol Sci, 352:1837-43. 